\title{
A one-trial non-shock passive avoldance task for rats
}

\author{
RICHARD W. THOMPSON and RICHARD A. GALOSY \\ WESTERN WASHINGTON STATE COLLEGE, Bellingham, \\ Washington 98225
}

Apparatus and procedure are described for providing a one-trial nonshock passive-avoidance task.

Current experiments employing one-trial locomotor passiveavoidance tasks have uniformly used shock as the noxious stimulus. Use of only one form of noxious stimuli in these one-trial passive-avoidance tasks seriously restricts the generalizations that can be drawn. This restriction applies particularly to recent investigations of the physiological bases of memory (consolidation theory, for example). The apparatus and procedure described in this paper provide a one-trial nonshock passive-avoidance task that affords a means of extending the generality of the data obtained in passive-avoidance situations.

\section{METHOD}

\section{Apparatus}

A diagram of the apparatus is presented in Fig. 1. The covered box is painted flat black except for the floor and platform, which are painted gray. The floor, divided in half and hinged at each side to act as a trap-door, is supported at the back by a piece of plastic attached to a solenoid. Activation of the solenoid withdraws the support for the floor. The bottom of the box is filled to a depth of 4 in. with ice water $(3 \mathrm{deg} C)$. The light above the platform is a $6-\mathrm{W}, 110 \mathrm{~V}$ bulb mounted in a reflector. The box is placed on a table 31 in. above the floor so that the platform extends out into space away from any wall. The experimental room is lighted by a $25-\mathrm{W}$ red bulb 48 in. above the apparatus.

\section{Procedure}

The Ss are tested on four consecutive days. On Days 1, 3, and 4 , the Ss are given three trials with an ITI of at least $60 \mathrm{sec}$. On each trial $S$ is placed on the platform facing the hole in the wall, and step-through latency (STL) is measured from the time of placement until the base of S's tail enters the box. The $S$ is left in the box for $2 \mathrm{sec}$. (The apparatus could be automated by mounting the platform on a microswitch that starts a clock when the $S$ is placed on the platform. A photocell mounted 1 in. above the trap-door floor and 6 in. from the entrance could be wired through a relay to stop the clock and activate the solenoid.) On Day 2 control Ss are given a single trial like those of Day 1, but experimental Ss are dropped as soon as they enter the box. After the single trial on Day 2, all Ss are placed in a $12 \times 10 \times 30 \mathrm{in}$. holding cage for $5 \mathrm{~min}$. A $250 \cdot \mathrm{W}, 110 \mathrm{~V}$ infrared heat lamp is suspended $17 \mathrm{in}$. above one end of the holding cage to warm and dry the Ss.

\section{Supporting Data}

Two experiments using Sprague-Dawley female albino rats, 90 days of age, have been conducted using the procedure described above. In Experiment 1, median STL for Days 1, 2, 3, and 4 for control Ss $(\mathrm{N}=10)$ were $4.9,3.2,9.7$, and $17.2 \mathrm{sec}$, respectively, and for experimental $\mathrm{Ss}(\mathrm{N}=9)$ were $6.1,4.2,87.5$, and $52.7 \mathrm{sec}$, respectively. There were no differences in latencies between control and experimental groups on Days 1 and 2, but the differences between the two groups on Days 3 and 4 were

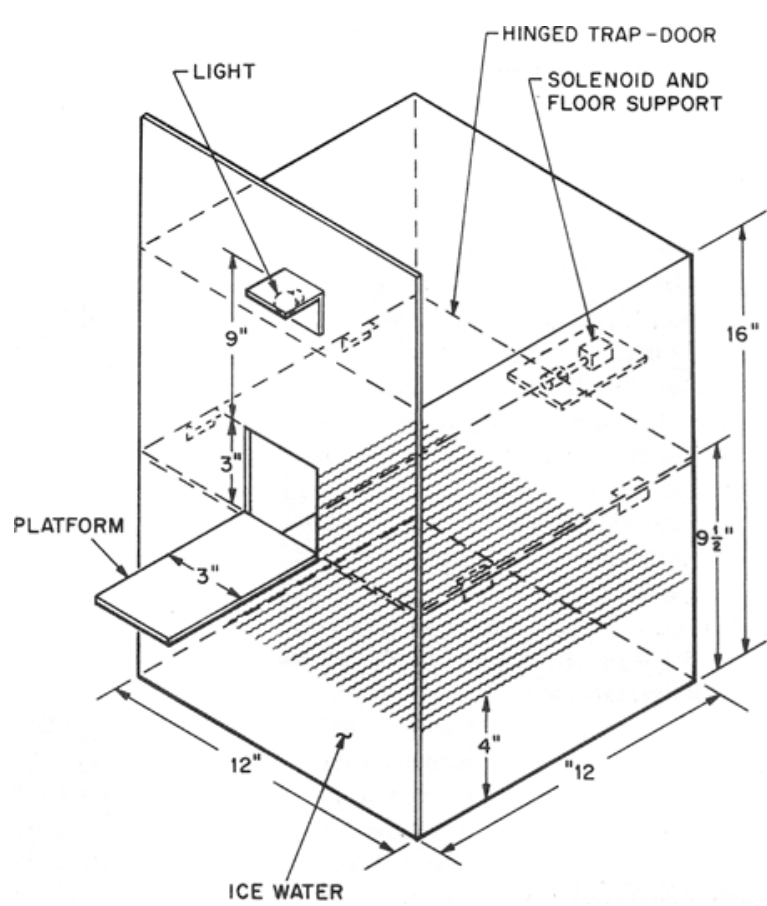

Fig. 1. Diagram of the apparatus.

significant (Mann-Whitney $U$ test, $p<.05$ ). Both control and experimental Ss showed significant increases in STL from Day 1 to Days 3 and 4 (Wilcoxon test, $p<.02$ ). The change from Day 3 to Day 4 was not significant for either group. Although both groups showed significant increases from Day 1 to Days 3 to 4 , the increase for the experimental Ss was much greater than for the controls. The behavior on the platform differed markedly for the $S s$ in the two groups. Control Ss engaged in visual exploration, which increased their STL, whereas the experimental Ss showed the typical crouching and freezing of fearful rats.

In Experiment 2, median STL in seconds for control $(\mathrm{N}=9)$ and experimental groups $(\mathrm{N}=9)$ for Days $1,2,3$, and 4 , respectively, were: controls-9.3, 2.7, 19.2, and 10.2; experimentals $-5.9,1.6,180.0$ (maximum time for Ss on platform was set at $180.00 \mathrm{sec}$ ), and 70.3 . There were no differences between the two groups on Days 1 and 2, but experimental Ss had significantly longer latencies on Day $3(\mathrm{p}<.02)$ and approached significance on Day $4(p<.10)$. There were no significant changes across days for control Ss, but the experimentals showed significant increases from Day 1 to Day 3 and from Day 1 to Day $4(\mathrm{p}<.01)$. The decrease from Day 3 to Day 4 was also significant $(p<.02)$. The results of the two experiments are consistent and indicate acquisition and retention of the passive-avoidance response.

The step-through drop task is very similar to the step-through shock passive-avoidance task and appears to be as efficient and effective. Whether all three forms of noxious stimulation (falling, forced swimming, and ice water) are necessary to produce learning cannot be specified at this time. These stimuli do have the advantage of being more ecologically normal than electric shock, and the task can be adapted for many different species. 